TRANSACTIONS OF THE

AMERICAN MATHEMATICAL SOCIETY

Volume 195, 1974

\title{
FIELDS OF CONSTANTS OF INTEGRAL DERIVATIONS ON A $p$-ADIC FIELD
}

BY

HENRY W. THWING AND NICKOLAS HEEREMA

ABSTRACT. Let $K_{0}$ be a $p$-adic subfield of a $p$-adic field $K$ with residue fields $k_{0} \subset k$. If $K_{0}$ is algebraically closed in $K$ and $k$ is finitely generated over $k_{0}$ then $K_{0}$ is the subfield of constants of an analytic derivation on $K$ or equivalently, $K_{0}$ is the in variant subfield of an inertial automorphism of $K$. If (1) $k_{0}$ is separably algebraically closed in $k$, (2) $\left[k_{0}^{p-1} \cap k: k_{0}\right]<\infty$ and (3) $k$ is not al gebraic over $k_{0}$ then there exists a p-adic subfield $K_{0}$ over $k_{0}$ which is algebraically closed in $K$. All subfields over $k_{0}$ are algebraically closed in $K$ if and only if $k_{0}$ is al gebraically closed in $k$. Every derivation on $k$ trivial on $k_{0}$ lifts to a derivation on $K$ trivial on $K_{0}$ if $k$ is separable over $k_{0}$. If $k$ is finitely generated over $k_{0}$ the separability condition is necessary. Applications are made to invariant fields of groups of inertial automorphisms on p-adic fields and of their ramification groups.

I. Introduction. Let $d$ be a derivation on a field $K$ having characteristic zero. It is well known that the field of constants $K_{0}$ of $d$ is algebraically closed in $K$. Conversely R. Baer showed in 1927 [1] that given any subfield $K_{0}$ of $K$ algebraically closed in $K$ there is a derivation on $K$ having $K_{0}$ as field of constants.

If $K$ is a $p$-adic field and $d$ is an analytic derivation on $K$, that is, $d$ is continuous in the $p$-adic topology, then the field of constants $K_{0}$ of $d$ is a $p$-adic subfield containing the inertial subfield $K^{*}$. Thus, generally, in this paper $K \supset$ $K_{0} \supset K^{*}$ will be $p$-adic fields with residue fields $k \supset k_{0} \supset k^{*}, k^{*}$ being the maximal perfect subfield of $k$. The main result of $\$$ II states that if $K_{0}$ is a $p$-adic subfield of $K$, algebraically closed in $K$ and $k$ is finitely generated over $k_{0}$ then $K_{0}$ is the field of constants of an analytic derivation on $K$ (Theorem 2.6). For convenience of language we will call a field of constants of an analytic derivation on $K$ simply a constant subfield, or constant field.

In $\$$ III we investigate which subfields $k_{0}$ of $k$ are residue fields of constant subfields of $K$ by determining which $k_{0}$ have $p$-adic fields over them which are algebraically closed in $K$. The principal result in this direction (Theorems 3.5

Received by the editors November 16, 1972.

AMS (MOS) subject classifications (1970). Primary 12B15, 13B10; Secondary 12F 15.

Key words and phrases. Analytic derivation, integral derivation, field of constants, p-adic field, relative algebraic closure. 
and 3.6) states that if (1) $k_{0}$ is separably algebraically closed in $k_{0}(2)\left[k_{0}^{p-1} n\right.$ $\left.k: k_{0}\right]<\infty$, and (3) $k$ is not algebraic over $k_{0}$ then there exists a $p$-adic subfield $K_{0}$ algebraically closed in $K$ having $k_{0}$ as residue field. All $p$-adic subfields over $k_{0}$ are algebraically closed in $K$ if and only if $k_{0}$ is algebraically closed in $k$. Results of $\$ \oint$ II and III combine to indicate, assuming that $k$ is finitely generated over $k_{0}$, exactly when there is at least one constant subfield over $k_{0}$ and the circumstances under which all $p$-adic subfields over $k_{0}$ are constant subfields. The conditions are, respectively, $k_{0}$ separably algebraically closed in $k$ and $k_{0}$ algebraically closed in $k$ (Theorem 3.10).

Example 1 of the Appendix exhibits a subfield $k_{0}$ algebraically closed in $k$ and two $p$-adic subfields $K_{0}$ and $K_{0}^{\prime}$ each having $k_{0}$ as residue field such that $K_{0}$ is a constant subfield and $K_{0}^{\prime}$ is not. Needless to say $k$ is not finitely generated over $k_{0}$.

In Example 2 a subfield $k_{0}$ of a field $k$ of characteristic $p$ is constructed such that $k_{0}$ is algebraically closed in $k$ and the maximal perfect subfield of $k$ is contained in $k_{0}$. However, if $K$ is a $p$-adic field with residue field $k$ and $K_{0}$ is any $p$-adic subfield $w$ ith residue field $k_{0}$ the only analytic derivation on $K$ whose constant field contains $K_{0}$ is the zero derivation. Thus, though $K_{0}$ is algebraically closed in $K$ and $K^{*} \subset K_{0}, K_{0}$ is not the field of constants of any set of analytic derivations.

§IV extends results of Heerema on lifting derivations on $k$ to $K$. In [4] it is shown that every derivation on $k$ lifts to a derivation on $K$. Theorem 4.1 addresses the question: Under what circumstances will all derivations on $k$ trivial on $k_{0}$ lift to a derivation on $K$ trivial on $K_{0}$ ? The result is that if $k$ is separable over $k_{0}$ every derivation on $k$ over $k_{0}$ ( $k_{0}$ in the field of constants) lifts to a derivation on $K$ over $K_{0}$. The converse also holds if $k$ is finitely generated over $k_{0}$. An example (Example 3 of the Appendix) is provided which illustrates that the converse need not hold if $k$ is not finitely generated over $k_{0}$.

The relationship between the results discussed above and inertial automorphisms on $K$ is treated in $\$ \mathrm{~V}$. The group $G_{0}$ of inertial automorphisms of a $p$-adic field $K$ was first studied in 1939 by S. Mac Lane [9]. He defined the ramification series of $G_{0}$, determined the factors of successive terms of the ramification series and showed that $K^{*}$, the inertial subfield of $K$ is the invariant subfield of $G_{0}$ as well as of each term of the ramification series. Using derivations some of Mac Lane's work has been generalized to ramified extensions of $p$-adic fields by Heerema [2] - [5], Neggers [11], and Peter [12]. Heerema has noted that the inertial automorphisms of $K$ are all of the form $\exp (p d)$ where $d$ is an integral derivation on $K$ ( $d$ maps integers onto integers), and $p$ is the characteristic of the residue field of $K$ [2]. If $p \neq 2$ the field of constants of $d$ is also the invariant subfield of $\exp (p d)$, that is, $d(a)=0$ if and only if 
$\exp (p d)(a)=a$. Thus if $K_{0}$ is a subfield of $K$ with residue fields $k \supset k_{0}$ and $k$ is finitely generated over $k_{0}$ then the intermediate subfields of $K$ which are invariant fields for groups of inertial automorphisms are precisely the fields of constants of analytic derivations. These in turn are the intermediate $p$-adic subfields which are algebraically closed in $K$. This is contained in Theorem 5.2. Theorem 5.2 also contains an extension of a result of Mac Lane's referred to above: If $L$ is the group of all inertial automorphisms with invariant subfield $K_{1}$ then $K_{1}$ is the invariant field of each term of the ramification series of $L$.

II. Subfields of constants. We assume a familiarity with the subject of derivations on fields to the extent covered in Jacobson [7, Chapter IV]. Given a field $K$ and a subfield $K_{0}$ algebraically closed in $K, \mathrm{R}$. Baer has shown [1] that a derivation $d$ on $K$ having $K_{0}$ as field of constants can be constructed as follows.

Case 1. Assume first that the transcendency degree of $K$ over $K_{0}$ is finite and let $\left\{a_{1}, \cdots, a_{n}\right\}$ be a transcendency basis for $K$ over $K_{0}$. The conditions $d\left(a_{1}\right)=1$, and $d\left(a_{i}\right)=a_{i-1}^{-1}$ for $i=2, \cdots n$ uniquely determine a derivation on $K$ having $K_{0}$ as field of constants.

Case 2. Let $T=\left\{a_{a}\right\}$ be a transcendency basis for $K$ over $K_{0}$ well ordered with ordinal $\gamma$ which we can assume to be a limit ordinal. Let $d_{0}$ be the zero derivation on $K_{0}$ and let $K_{\alpha}=K_{0}\left(\left\{a_{\beta}\right\}_{\beta \leq \alpha}\right)$. For each $\alpha<\gamma, d_{\alpha}$ is defined to be the derivation on $K_{\alpha}$ into $K_{\alpha+1}$ which extends $d_{\rho}$ for $\rho<\alpha$ and such that $d_{\alpha}\left(a_{x}\right)=a_{x+1}$. This defines a derivation on $K_{0}(T)$ whose unique extension to $K$ is the desired derivation.

We indicate the proof of the following known fact $[1$, Corollary 1, p. 20] for the reader's convenience.

2.0 Lemma. Let $d$ be a derivation on a field $K$ with field of constants $K_{0}$. Let $u$ be separably algebraic over $K$ and let $d_{1}$ be the unique extensions of $d$ to $K(u)$. Then $u$ is $a d_{1}$ constant if and only if $u$ is separably algebraic over $K_{0}$.

We prove the necessity only. Let $g(X)=\Sigma a_{i} X^{i}$ be the minimum polynomial of $u$ over $K$. With $g^{d}(X)=\Sigma d\left(a_{i}\right) X^{i}$ and $g^{\prime}(X)=\Sigma i a_{i} X^{i-1}$ we have $g^{d}(u)+$ $g^{\prime}(u) d_{1}(u)=0$. Since $g^{\prime}(u) \neq 0, d_{1}(u)=0$ only if $g^{d}(u)=0$ or only if $g^{d}(X)=0$, that is only if $g(X) \in K_{0}[X]$.

Henceforth $K$ will represent a $p$-adic field with ring of integers $R$, and residue field $k$ having characteristic $p$. Also $H: K \rightarrow k$ will denote the natural place. A derivation $d$ on $K$ is analytic, or continuous in the p-adic topology if $d(R) C$ $p^{-n} R$ for some integer $n$. If $d(R) \subset R, d$ is an integral derivation. Let $\operatorname{Der}(K)$ denote the $K$ space of analytic derivations on $K$. Thus if $d \in \operatorname{Der}(K)$ then for some $n, p^{n} d$ is integral. 
The field of constants of $d \in \operatorname{Der}(K)$ is clearly closed in the $p$-adic topology and is thus a $p$-adic subfield. We have

2.1 Proposition. The field of constants $K_{0}$ of $d$ in $\operatorname{Der}(K)$ is a p-adic subfield of $K$ algebraically closed in $K$.

For the following lemma let $K_{2}$ be a field having a $p$-adic valuation, ring of integers $R_{2}$ and residue field $k_{2}$. Let $K_{1}$ be a subfield of $K_{2}$ with residue field $k_{1}$. Assume next that $U$ is a set of units in $K_{2}$ whose residues are algebraically independent over $k_{1}$ ( $U$ is a set of units in the valuation ring of $K_{2}$ ).

2.2 Lemma. If $d$ is a derivation mapping $K_{1}(U)$ into $K_{2}$ such that $d\left(K_{1} \cap R_{2}\right) \subset R_{2}$ and $d(U) \subset R_{2}$ then $d\left(K_{1}(U) \cap R_{2}\right) \subset R_{2}$.

Proof. An element $a$ in $K_{1}(U) \cap R_{2}$ has the rational form $f\left(u_{1} \cdots u_{n}\right) g\left(u_{1} \cdots u_{n}\right)^{-1}$, the $u_{i}$ being in $U$. Also, $f\left(X_{1} \ldots X_{n}\right)$ and $g\left(X_{1} \ldots X_{n}\right)$ have integer coefficients and at least one coefficient of $g\left(X_{1} \ldots X_{n}\right)$ is a unit. Then $g\left(u_{1} \cdots u_{n}\right)$ is a unit since $H\left(u_{1}\right), \ldots, H\left(u_{n}\right)$ are algebraically independent over $k_{1}$. Now $d\left(f\left(u_{1} \cdots u_{n}\right) \in R_{2}, d\left(g\left(u_{1} \cdots u_{n}\right)\right) \in R_{2}\right.$ and hence $d\left(f\left(u_{1} \cdots u_{n}\right) g\left(u_{1} \cdots u_{n}\right)^{-1}\right) \in R_{2}$.

We now assume $K_{2} \supset K_{1}$ as above and that $K_{1}$ is the field of constants of an integral derivation $d$ on $K_{2}$.

2.3 Lemma. The derivation $d$ extends uniquely to an integral derivation $d^{*}$ on the completion $K_{2}^{*}$ of $K_{2}$. The field of constants of $d^{*}$ is the closure $K_{1}^{*}$ of $K_{1}$ in $K_{2}^{*}$.

We show only that if $d^{*}(b)=0$ for a unit $b$ in $K_{2}$ then $b \in K_{1}^{*}$.

Let $b=\Sigma\left\{p^{i} b_{i} \mid b_{i} \in R_{2}, i=0,1 \ldots\right\}$. Since $d^{*}(b)=0$ we have $d\left(b_{0}\right) \epsilon$ $p R_{2}$ or $d\left(b_{0}\right)=p a_{0}, a_{0} \in R_{2}$, and $p d\left(b_{1}\right)+p\left(b_{0}\right) \in p^{2} R_{2}$ or $p d\left(b_{1}\right)+p a_{0} \in p^{2} R_{2}$. Hence $d\left(b_{1}\right)=-a_{0}+p a_{1} \in R_{2}$ and $p d\left(b_{1}\right)+d\left(b_{0}\right)=p^{2} a_{1}$. Proceeding inductively, we have $d\left(b_{i}\right)=-a_{i-1}+p a_{i}$ with $a_{i-1}$ and $a_{i}$ in $R_{2}$ and $d\left(p^{i} b_{i}+\cdots+b_{0}\right)=$ $p^{i+1} a_{i}$. Since $d\left(p^{i+1} b_{i+1}+\cdots+b_{0}\right)=p^{i+1} d\left(b_{i+1}\right)+p^{i+1} a_{i} \in p^{i+2} R_{2}$, $d\left(b_{i+1}\right)=-a_{i}+p a_{i+1}$ for $a_{i+1}$ in $R_{2}$. If $f_{i}=p^{-(i+1)} \sum\left\{p^{j} b_{j} \mid j=0, \ldots, i\right\}$ then $d\left(f_{i}\right)=a_{i}$ for $i \geq 0$ and hence $c_{0}=b_{0}-p f_{0}$ as well as $c_{i}=b_{i}+f_{i-1}-p f_{i}$ are all $d$ constants. That is $c_{i} \in K_{1}$ for $i \geq 0$. But $b=\Sigma p^{i} b_{i}=c_{0}+p f_{0}+$ $\Sigma\left(-f_{i-1}+c_{i}+p f_{i}\right) p^{i}=\Sigma_{i} c_{i} p^{i}$. Thus $b \in K_{1}^{*}$.

For the following theorem we assume $K_{0}$ to be a subfield of a $p$-adic field $K$ the residue fields being $k_{0} \subset k$.

2.4 Theorem. If $K_{0}$ is the field of constants of an analytic derivation on $K$ then $K_{0}$ is a p-adic subfield algebraically closed in $K$. Conversely, if $K_{0}$ is 
a p-adic subfield of $K$ algebraically closed in $K$ and (a) $k$ is finitely generated over $k_{0}$ then $K_{0}$ is the constant field of an analytic derivation on $K$.

Example 1 in the Appendix shows that condition (a) is not necessary. It also indicates that there are no necessary and sufficient conditions on the residue field only. It also demonstrates that $k_{0}$ need not be algebraically closed in $k$ (see Theorem 3.10).

Proof. The first sentence is Proposition 2.1. Proceeding to the converse, let $T$ be a set of representatives in $K$ of a transcendency basis $\bar{T}$ of $k$ over $k_{0}$. Define $d_{1}$ on $K_{0}(T)$ having $K_{0}$ as field of constants according to the prescription given at the beginning of this section. By Lemma $2.2 d_{1}$ is integral. The extension $d_{1}^{*}$ of $d_{1}$ to the closure $K_{0}(T)^{*}$ of $K_{0}(T)$ in $K$ also has $K_{0}$ as field of constants by Lemma 2.3. Since $k$ is finitely generated over $k_{0}$, [ $k$ : $\left.k_{0}(\bar{T})\right]<\infty$. Hence $\left[K: K_{0}(T)^{*}\right]<\infty$. Let $u$ be a primitive element for $K$ over $K_{0}(T)^{*}$ having minimum function $f(X)$. Denoting the extension of $d_{1}^{*}$ to $K$ by $d$, we have $d(u)=f^{d}(u) / f^{\prime}(u)$ which is in $p^{-n} R$ where $n=V\left(f^{\prime}(u)\right)$. Hence $d$ is analytic. By Lemma 2.0 the field of constants of $d$ is $K_{0}$.

Using the methods of $\$$ III one can also prove that if instead of condition (a) it is assumed that $k$ has a transcendency basis $\bar{T}$ over $k_{0}$ such that $k$ has bounded exponent over $k_{0}(\bar{T})$ then, retaining the assumption on $K_{0}$, there is a $p$-adic subfield $K_{0}^{\prime}$ with residue field $k_{0}$ which is the field of constants of an analytic derivation on $K$. Since this result is essentially independent of the rest of the paper we do not prove it. It does, however, relate to Example 1 of the Appendix.

For fields of characteristic zero the subfields which are constant fields of higher derivations (of finite or infinite rank) are precisely the fields of constants of derivations. This follows from the fact that if $\left\{D_{i}\right\}$ is a higher derivation on such a field $M$, then there is a sequence $\left\{d_{i}\right\}$ of derivations on $M$ such that

$$
D_{j}=\sum\left\{d_{i}, \cdots, d_{i_{r}} / r ! \mid i_{1}+\cdots+i_{r}=j\right\}
$$

for all $j\left[4\right.$, Theorem 3]. One sees directly that if $\left\{D_{i}\right\}$ is an analytic higher derivation on a $p$-adic field; that is, each $D_{i}$ is continuous, then the derivations of (2.5) are also analytic and conversely. The fields of constants of $\left\{D_{i}\right\}$ is the intersection of the field of constants of $d_{i}, i=1, \ldots, r$, where $r=$ rank $D_{i}(r \leq \infty)$. Since the intersection of any number of $p$-adic subfields of $K$ algebraically closed in $K$ is a $p$-adic subfield algebraically closed in $K$ we can restate Theorem 2.4 replacing analytic derivation with analytic higher derivations as follows. 
2.6 Theorem. If $K_{0}$ is the field of constants of an analytic bigher derivation on $K$ then $K_{0}$ is a p-adic subfield of $K$ algebraically closed in $K$. Conversely, if $K_{0}$ is a p-adic subfield of $K$ algebraically closed in $K$ and (a) $k$ is finitely generated over $k_{0}$ then $K_{0}$ is the constant field of an analytic bigher derivation.

Proof. To prove the converse we simply take an analytic derivation $d$ on $K$ having $K_{0}$ as field of constants. The rank $r$ higher derivation $\left\{D_{i} \mid i=0, \ldots, r\right\}$ where $D_{i}=d^{i} / i$ ! has field of constants $K_{0}$.

III. Residue fields of constant subfields. As the heading suggests we consider in this section those subfields $k_{0}$ of the residue field $k$ of a $p$-adic field $K$ which are residue fields of constant fields, or more generally, residue fields of $p$-adic subfields algebraically closed in $K$ and containing the inertial subfield $K^{*}$.

3.1 Theorem. Every p-adic subfield $K_{0}$ with $K^{*} \subset K_{0} \subset K$ is algebraically closed in $K$ if and only if $k_{0}$, the residue field of $K_{0}$, is algebraically closed in $k$.

Proof. If $K_{0}$ is not algebraically closed in $K$, choose $u$ in $K \backslash K_{0}$, algebraic over $K_{0} . k_{0}$ is not the residue field of $K_{0}(u)$ [9, Lemma 4, p. 430] so there exists a unit $v$ in $K_{0}(u)$ with residue $H(v)$ not in $k_{0}$. If $\Sigma a_{i} X^{i}$ is the minimum polynomial of $v$ over $K_{0}$, then $H(v)$ satisfies the polynomial $\Sigma H\left(a_{i}\right) X^{i}$ in $k_{0}[X]$ and $k_{0}$ is not algebraically closed in $k_{\text {. }}$

Converesly, if $k_{0}$ is not algebraically closed in $k$, the $p$-adic field $K^{\prime} \supset K_{0}$ may be constructed using standard methods [4, pp. 377-378] having $k$ as residue field and such that $K_{0}$ is not algebraically closed in $K^{\prime}$. There is an analytic isomorphism from $K^{\prime}$ onto $K$ [9, Corollary 1, p. 431]. Taking $K_{0}^{\prime}$ as the image of $K_{0}$ under this map, $K_{0}^{\prime}$ has residue field $k_{0}$ and is not algebraically closed in $K$.

If $K_{0} \subset K$ is algebraically closed in $K$, Hensel's lemma yields easily that $k_{0}$ is separably algebraically closed in $k$. In the following we develop a partial converse of this statement.

3.2 Lemma. Suppose $D$ is a derivation on a field $K$ of characteristic zero with field of constants $K_{0}$. Then the equation $D(y)+(i / m)[D(b) / b] y=0$, where $b \in K, b \neq 0$, and $i$ and $m$ are positive rational integers, bas a nontrivial solution for $y \in K$ if and only if $b^{i}$ is of the form $a c^{m}$ for some $a \in K_{0}$ and $c \in K$.

Proof is by showing that $c^{-1}$ solves the equation in one case and, conversely, if $c^{-1}$ solves the equation, then $D\left(b^{i}\right) / b^{i}=D\left(c^{m}\right) / c^{m}$ so $b^{i}$ and $c^{m}$ differ by a factor which is a $D$-constant. 
3.3 Lemma. Suppose $K_{0} \subset K_{1}$ are fields of cbaracteristic zero, $K_{0}$ algebraically closed in $K_{1}$. Let $u$ be algebraic over $K_{1}$ with minimum polynomial $f(X)=X^{m}-b$. Then $K_{0}$ is algebraically closed in $K_{1}(u)$ if and only if $b^{i}$ is not of the form ac with $a$ in $K_{0}, c$ in $K_{1}$, for $i=1,2, \ldots, m-1$.

Proof. By Baer [1, Theorem 5, p. 24], there exists a derivation $D$ on $K_{1}$ with field of constants $K_{0}$. $D$ extends to a derivation $D^{*}$ on $K_{1}(u)$ with $D^{*}(u)=$ $D(b) / m u^{m-1}$. It suffices to show that $K_{0}$ is the field of constants of $D^{*}$. Letting $c=\Sigma_{i=0}^{m-1} c_{i} u^{i}, c_{i} \in K_{1}$, be a $D^{*}$-constant, linear independence of $\left\{1, u, u^{2}\right.$, $\left.\cdots, u^{m-1}\right\}$ yields the equations:

$$
\begin{aligned}
D\left(c_{0}\right) & =0 \quad \text { so } c_{0} \in K_{0}, \\
D\left(c_{i}\right) b+(i / m) c_{i} D(b) & =0, \quad i=1,2, \cdots, m-1 .
\end{aligned}
$$

The result follows from Lemma 3.2.

The following simplify the use of Lemma 3.3.

3.4 Lemma. Let $K_{0} \subset K_{1}$ be fields. If $b \in K_{1}$ is sucb that $b^{i}=a c^{m}$ for some $a \in K_{0}, c \in K_{1}, 0<i<m$, and $i$ is the smallest sucb integer for $b^{i}$ to bave this form, then $i$ divides $m$.

Proof. b8.c.d.(i, $m)$ has the form $a c^{m}, a$ in $K_{0}$, if $b^{i}$ does.

3.5 Corollary. Under the bypotheses of Lemma 3.4, if $m=p^{n}$ where $p$ is a prime, then $i$ is a power of $p$.

3.6 Theorem. Let $K$ be a p-adic field with residue field $k_{0}$ Suppose $k_{0} C$ $k$ is sucb that:

(1) $k_{0}$ is separably algebraically closed in $k_{\text {, }}$

(2) $\left[k_{0}^{p-1} \cap k: k_{0}\right]<\infty$, and

(3) $\operatorname{trans} \operatorname{deg} k / k_{0} \neq 0$.

Then there exists some p-adjc subfield $K_{0} \subset K$ with residue field $k_{0}$ such that $K_{0}$ is algebraically closed in $K$. All such $K_{0}$ are algebraically closed in $K$ if and only if $k_{0}$ is algebraically closed in $k_{\text {. }}$

Define the function $\phi_{K_{0}, K}: k_{0} \cap k^{p} \backslash\{0\} \rightarrow k^{+} / k_{0}^{+}$(the additive groups of $k$ and $k_{0}$ ) as follows: For any $x \in k_{0} \cap k^{p}$, let $y=x^{p-1}$. Let $c \in K_{0}$ and $b \in K$ be such that $H(c)=x^{-1}$ and $H(b)=y$. Note that $1=c b^{p}$, $\bmod p R$. Define

$$
\phi_{K_{0}, K}(x)=H\left(\left(1-c b^{p}\right) / p\right)+k_{0}^{+}
$$

$\phi=\phi_{K_{0}, K}$ is seen to be well defined. It is easily seen that if $k_{0} \subset k_{1} \subset k$ with $p$-adic over fields $K_{0} \subset K_{1} \subset K$ then $\phi_{K_{0, K}}=\left.\phi_{K_{0}, K}\right|_{k_{0}} \cap k_{1}^{p \cdot}$ 
3.7 Lemma. If $K_{0}$ is algebraically closed in $K$ and $u$ is a root of the irreducible polynomial $X^{p}-v(1+p t)$, with $v$ in $R \cap K_{0}$ and $t$ a unit in $K$ and if $H(t)$ is not in $\phi\left(k_{0} \cap k^{p}\right)$, then $K_{0}$ is algebraically closed in $K(u)$.

Proof. $K_{0}$ is algebraically closed in $K(u)$ unless $v(1+p t)=b^{p} / a$, with $a$ in $K_{0}, b$ in $K$ or, since $v \in K_{0}$, unless $(1+p t)=b^{p} / a$ with $a$ in $K_{0}, b$ in $K$. This follows from Lemma 3.3 and Corollary 3.5. We can assume $b$ and $a$ to be units. If $a(1+p t)=b^{p}$ then $H(a) \epsilon k_{0} \cap k^{p}$. Also, by definition of $\phi, \phi(a)=$ $H(-t)$ and the result follows.

3.8 Lemma. Let $k_{1} / k_{0}$ be separable (in the linearly disjoint sense) and let $U$ be a subset of an overfield such that $U^{p} \subset k_{0}$. If $v \in k_{1}(U)$ with $v^{p} \in k_{0}$, then $v \in k_{0}(U)$.

Proof. Note first that $k_{1}(U)=k_{1} \otimes_{k_{0}} k_{0}(U)$. Then if $\left\{y_{a}\right\}$ is a linear basis for $k_{1} / k_{0}$ with $1 \in\left\{y_{\alpha}\right\}$ and $\left\{x_{\beta}\right\}$ a linear basis for $k_{0}(U) / k_{0}$.

$$
v=\sum_{a} \sum_{\beta} w_{\beta a} z_{\beta} y_{a}, \quad w_{\beta a} \epsilon k_{0}, \quad v^{p}=\sum_{a}\left(\sum_{\beta} w_{\beta a}^{p} z_{\beta}^{p}\right) y_{a}^{p} \epsilon k_{0} .
$$

If none of the $y_{\alpha}$ in (3.9) is 1 , separability yields $v=0$. If $y_{\gamma}=1$ in (3.9), separability gives:

$$
v^{p}=\sum_{\beta} w_{\beta \gamma}^{p} z_{\beta}^{p}, \quad v=\sum_{\beta} w_{\beta \gamma} z_{\beta} \in k_{0}(U) .
$$

Proof of Theorem 3.6. Let $k_{1}$ be the subfield of $k$ obtained by first extending $k_{0}$ by a transcendency basis for $k / k_{0}$, and then taking the separable algebraic closufe of this field in $k_{\text {. Then }} k_{1}$ is separable over $k_{0}$ (in the linearly disjoint sense). $k_{0}$ is algebraically closed in $k_{1}$, and $k$ is purely inseparable over $k_{1}$. Let $U=\left\{u_{1}, u_{2}, \cdots, u_{n}\right\}$ be a $p$-basis for $k_{0}^{p^{-1}} \cap k$ over $k_{0}$. Let $k_{2}=k_{1}(U)$. Let $K_{0}^{\prime} \subset K_{1}$ be $p$-adic fields having $k_{0} \subset k_{1}$ as residue fields. By Theorem 3.1, $K_{0}^{\prime}$ is algebraically closed in $K_{1}$. Let $t_{1}$ be in $k_{1} \backslash k_{0}$ and choose $t$ in $K_{1}$ so that $H(t)=t_{1}$. Since $k_{0}$ is algebraically closed in $k_{1}, \phi$ is trivial on $k_{0} \cap k_{1}^{p}$ so $t_{1}^{(n+1) !}+k_{0}^{+}$is not in $\phi\left(k_{0} \cap k_{1}^{p}\right)$. Let $V=\left\{v_{1}, \cdots, v_{n}\right\}$. $C K_{0}^{\prime}$ be such that $H\left(v_{i}\right)=u_{i}^{p}$. Using Mac Lane's construction procedure [8, pp. $377-378]$ let $K_{1}=K_{1,0}$ and $K_{1, i}=K_{1, i-1}\left(w_{i}\right)$ where $w_{i}$ is a root of $x^{p}-$ $v_{i}\left(1+p t^{(n-i+2) !}\right)$, and let $K_{2}=K_{1, n} \cdot K_{1, i}$ has residue field $k_{1}\left(u_{1}, \cdots, u_{i}\right)$. We wish to show that $K_{\rho}^{\prime}$ is algebraically closed in $K_{2}$ by observing that for $i=1, \cdots, n, t_{1}^{(n-i+1) ?}$ is not in $\phi\left[k_{0} \cap\left[k_{1}\left(u_{1}, \cdots, u_{i}\right)\right]^{p}\right]$ and using Lemma 3.8. Let $x \in k_{0}, y \in k_{1}\left(u_{1}, \cdots, u_{i}\right)$ where $y^{p}=x^{-1}$. By Lemma 3.8, $y$ is in $K_{0}\left(u_{1}, \cdots, u_{i}\right)$. Choose $X \in K_{0}$ and $Y \in K_{0}\left(w_{1}, \cdots, w_{i}\right)$ so that $H(X)=x$, $H(Y)=y$. By definition of the $w_{i}$ 's, $H\left(Y^{p}\right) \in k_{0}\left(t_{1}^{(n-i+2) !}, u_{1}, \cdots, u_{i}\right)$ and hence $\phi(x) \in k_{0}\left(t_{1}^{(n-i+2) !}, u_{1}, \cdots, u_{i}\right)$. Thus $t_{1}^{(n-i+1) !}$ is not in $\phi\left[k_{0} \cap\left(k_{1}\left(u_{1}, \cdots, u_{i}\right)\right)^{p}\right]$. It follows that $K_{0}^{\prime}$ is algebraically closed in $K_{2}$ 
and $t_{1} \notin\left[k_{0} \cap k_{2}^{p}\right]=\left[k_{0} \cap k^{p}\right]$.

It follows from this fact and the proof of Lemma 3.7 that if $K_{3}$ is any $p$-adic field with residue field $k_{3}$ such that $K_{2} \subset K_{3}$ and $k_{2} \subset k_{3} \subset k_{\text {, then }} 1+p t$ is not of the form $a c^{p}$ for any $a \in K_{0}^{\prime}$ and $c \in K_{3} . K^{\prime}$ is constructed over $K_{2}$ by Mac Lane's procedure, well-ordering a set of generators for $k / k_{2}$ such that each extension is by a pth root. At each step, if the corresponding polynomial over the $p$-adic field $K_{a}$ at that stage is $x^{p}-b_{\alpha}$ with $b_{\alpha}$ of the form $a c^{p}, a \in K_{0}^{\prime}$ and $c \epsilon$ $K_{a}$, a second choice, $b_{a}(1+p t)$ is not of this form and the polynomial $x^{p}-b_{\alpha}(1+p t)$ is to be used instead. By transfinite induction $K_{0}^{\prime}$ is seen to be algebraically closed in $K^{\prime}$. There exists an analytic isomorphism from $K^{\prime}$ onto $K[9$, Corollary 1, p. 431]. Letting $K_{0}$ be the image of $K_{0}^{\prime}$ under this isomorphism establishes the result.

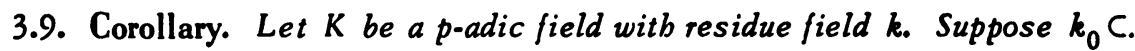
$k$ is sucb that

(1) $k$ is finitely generated over $k_{0}$,

(2) trans deg $k / k_{0} \neq 0$.

Then there exists some p-adic subfield $K_{0} \subset K$ with residue field $k_{0}$ sucb that $K_{0}$ is algebraically closed in $K$ if and only if $k_{0}$ is separably algebraically

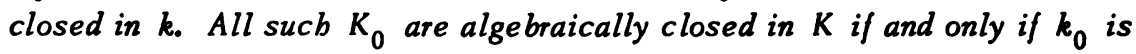
algebraically closed in $k$.

Combining Corollary 3.9 and Theorem 2.4 we have

3.10 Theorem. Let $K$ be a p-adic field with residue field $k$ and let $k_{0}$ be a subfield of $k$ sucb that $k$ is finitely generated over $k_{0}$ but is not algebraic over $k_{0}$. Then there exists some p-adic subfield $K_{0} \subset K$ with residue field $k_{0}$ such that $K_{0}$ is the constant field of an analytic derivation if and only if $k_{0}$ is separably algebraically closed in $k$. All sucb $K_{0}$ are constant fields of analytic derivations if and only if $k_{0}$ is algebraically closed in $k_{\text {. }}$

Example 1 of the Appendix demonstrates that hypothesis (2) of Theorem 3.6 is not necessary for $K_{0}$ is algebraically closed in $K$ whereas $\bar{x}_{n}=\bar{t}^{p^{n}}-\bar{u}_{n}^{p^{n}}$, for $n \geq 1$, and so (2) fails.

IV. Lifting derivations. In this section we extend results of the second author on lifting derivations from $k$ to $K$. In particular, in 1962 [4] Heerema showed that all derivations on $k$ lift to derivations on $K$. The following generalization involves field of constant conditions on both fields. Let $\operatorname{Der}\left(k / k_{0}\right)$ be the subspace of those derivations on $k$ which restrict to the zero map on $k_{0}$. $\operatorname{Der}\left(K / K_{0}\right)$ is similarly defined. 
4.1 Theorem. Let $K \supset K_{0}$ be p-adic fields with residue fields $k \supset k_{0}$. If $k$ is separable over $k_{0}$ then every $\delta$ in $\operatorname{Der}\left(k / k_{0}\right)$ lifts to $d$ in $\operatorname{Der}\left(K / K_{0}\right)$. Conversely, if $k$ is finitely generated over $k_{0}$ and every $\delta$ in $\operatorname{Der}\left(k / k_{0}\right)$ lifts to $\operatorname{Der}\left(K / K_{0}\right)$ then $k$ is separable over $k_{0}$.

Example 2 of the Appendix shows that the converse need not obtain if $k$ is not finitely generated over $k_{0}$.

Proof. Let $\bar{S}$ be a $p$-basis for $k_{0}$. Since $k$ is separable over $k_{0}$ a $p$-basis for $k$ can be constructed by joining to $\bar{S}$ a $p$-basis $\bar{T}$ for $k$ over $k_{0}$. Choose a set of representatives $S$ for $\bar{S}$ from $K_{0}$ and a set $T$ of representatives of $\bar{T}$ from $K$. Each $\delta \in \operatorname{Der}\left(k / k_{0}\right)$ is determined by the set $\{\delta(\bar{t}) \mid \bar{t} \in \bar{T}\}$. Similarly each $d$ in $\operatorname{Der}(K)$ is determined by its action on a set of representatives of a $p$-basis for $k[6$, Theorem 4]. We define $d$ in $\operatorname{Der}(K)$ by the conditions $d(s)=$ 0 for $s \in S$ and $d(t)=a_{t}$, where $H\left(a_{t}\right)=\delta(\bar{t})$, for $t \in T$. Clearly $d$ is trivial on $K_{0}$ and $H d=\delta H$, that is, $d$ induces $\delta$.

Conversely, suppose $k$ to be finitely generated over $k_{0}$ but not separably generated. Then $\operatorname{Der}\left(k / k_{0}\right)$ has dimension over $k$ greater than the transcendency degree of $k$ over $k_{0}$ [7, Corollary, p. 179]. In constructing $K$ over $K_{0}$ by the procedure described in Mac Lane [8] $K_{0}$ is extended by an algebraically independent set $T$ of cardinality the transcendency degree of $k$ over $k_{0}$. By Lemma 2.3 , any integral derivation on $K$ over $K_{0}$ is uniquely determined by its action on $T$ since once it is defined on $T$ it extends uniquely to the closure of $K_{0}(T)$ and then to a separably algebraic extension. Therefore the dimension of the subspace of $\operatorname{Der}\left(k / k_{0}\right)$ induced by $\operatorname{Der}\left(K / K_{0}\right)$ is no more than the cardinality of $T$ which is less than the dimension of $\operatorname{Der}\left(k / k_{0}\right)$. The result follows.

V. Inertial automorphisms and analytic derivations. F. K. Schmidt proved in the early thirties that an automorphism $a$ on $2 p$-adic field $K$ had to be value preserving. Thus every $a$ induces an automorphism on the residue field $k$. If $a$ induces the identity map on $k, \alpha$ is an inertial automorphism. For $d$ an integral derivation on $K, \exp (p d)=1+p d+p^{2} d^{2} / 2 ! \ldots$ is an inertial automorphism. Heerema has shown that if $p \neq 2$ every inertial automorphism on $K$ has the form $\exp (p d)$ for $d$ an integral derivation on $K$ [2, Theorem 3.2].

\subsection{Proposition. Let $d$ be an integral derivation on a p-adic field $K$ with} $p \neq 2$. The invariant subfield of the inertial automorphism $\exp p d$ is the constant field of $d$.

Proof. Let $K_{0}$ be the constant field of $d, H$ the invariant field of $\exp (p d)$. Clearly $K_{0} \subset H$. Consider a unit $b$ in $H$. If $d(b) \neq 0$ then $d(b)=p^{r} u$ for some $r \geq 0$ and unit $u$ in $K$. Thus $V(p d(b)) x r+1$. We need only show that 
$V\left(p^{n} d^{n}(b) / n !\right)>r+1$ for $n>1$ to conclude that $\exp p d(b) \neq b$ contradicting the choice of $b$. Clearly, $V\left(p^{n} d^{n}(b) / n !\right)>r+1$ for $1<n<p$. By assumption $p \geq 3$. Since $V(n !) \leq(n-1) /(p-1)\left[2\right.$, Lemma 2.4, p. 301] we have $V\left(p^{n} d^{n}(b) / n !\right) \geq$ $n+r-(n-1) /(p-1)$. Also, $n-(n-1) /(p-1) \geq n-(n-1) / 2$ and $n-(n-1) / 2>1$ for $n \geq 3$. Hence $\exp p d(b)=b+p d(b)+\cdots \neq b$, a contradiction, so it must be that $d(b)=0$. Let $G_{0}$ be the group of inertial automorphisms on a $p$-adic field $K$. The ramification series on $K$ is the chain $\left\{G_{i}\right\}$ of invariant subgroups where $G_{i}=$ $\left\{a \in G_{0} \mid \alpha(a)=a, \bmod p^{i}\right.$, for $a$ an integer in $\left.K\right\}$. In 1939 Mac Lane showed that, for all $i \geq 0$, the invariant subfield of $G_{i}$ is the inertial subfield $K^{*}$ of $K$ [9, Theorem 16]. For any group $L$ of inertial automorphisms on $K$ let $g(L)$ be the subfield of $L$ invariants and for a subfield $K_{0}$ of $K$ let $\varrho_{(}\left(K_{0}\right)$ be the group of inertial automorphisms of $K$ leaving $K_{0}$ invariant. Define the ramification series of the group $L$ as above i.e., $L_{i}=\left\{a \in L \mid \alpha(a)=a, \bmod p^{i}\right.$, for $a$ an integer in K\}.

5.2. Theorem. Let $K \supset K_{0}$ be p-adic fields with residue fields $k \supset k_{0}$ and assume $k$ finitely generated over $k_{0}$. A field $K_{1}$ with $K \supseteq K_{1} \supseteq K_{0}$ is the in. variant subfield of a group of inertial automorphisms on $K$ if and only if $K_{1}$ is a p-adic subfield algebraically closed in $K$. If $L=\varrho\left(K^{\prime}\right)$ for some subfield $K^{\prime}$ of $K$ then $g(L)=g\left(L_{i}\right)$ for $i \geq 1$.

Proof. If $K_{1}=g(L)$ for a group $L$ of inertial automorphisms then $K_{1}$ is the constant field of a set of analytic derivations by Proposition 5.1 and is, thus, the intersection of $p$-adic subfields each of which is algebraically closed in $K$. Thus $K_{1}$ is a $p$-adic subfield algebraically closed in $K$. Conversely, if $K_{1}$ satisfies the conditions of the theorem then by Corollary 2.7, $K_{1}$ is the constant subfield of an integral derivation. By Proposition 5.1, $K_{1}=g\left(\varrho_{(}\left(K_{1}\right)\right)$.

The last sentence follows immediately from the fact that if $\exp (p d) \in L$ then $\exp \left(p^{i} d\right)$ is in $L_{i}$ and $\mathscr{T}(\exp p d)=\mathscr{I}\left(\exp p^{i} d\right)$.

\section{Appendix}

\section{Example 1.}

Summary. Two $p$-adic fields $K_{0}$ and $K_{0}^{\prime}$ are constructed in the same $p$-adic field $K$. They are both algebraically closed in $K$ and have the same residue field. $K_{0}$ is the field of constants of an integral derivation on $K$ while $K_{0}^{\prime}$ is not. Their common residue field $k_{0}$ is not algebraically closed in the residue field $k$ of $K$.

Construction. Let $k^{*}$ be a perfect field of characteristic $p \neq 0$. Let $\left\{\tau, \bar{x}_{1}\right.$, $\left.\bar{x}_{2}, \ldots\right\}$ be a denumerable set of quantities algebraically independent over $k^{*}$. For $n \geq 1$ let $\bar{u}_{n}$ be a root of $\bar{f}_{n}(z)=z^{p^{n}}-\left(\bar{x}_{n}+\bar{t}^{p^{n}}\right)$ over $k^{*}\left(\bar{t}_{1} \bar{x}_{1}, \bar{x}_{2}, \ldots\right)$. Define $k_{0}$ to be $k^{*}\left(\bar{x}_{1}, \bar{x}_{2}, \ldots\right)$ and $k=k_{0}\left(\bar{t}_{1} \bar{u}_{1}, \bar{u}_{2}, \ldots\right)$. Let $K_{0}$ be a 
$p$-adic field having $k_{0}$ as its residue field with representatives $x_{i}$ of $\bar{x}_{i}$. Extend $K_{0}$ by a transcendental $t$, representing $\bar{t}$, and complete $K_{0}(t)$ to obtain a $p$-adic field $K_{1}$. $K_{0}$ is algebraically closed in $K_{1}$.

Extensions $K_{1}^{\prime}$ and $K_{1}^{\prime \prime}$ are obtained by extending $K_{1}$ by roots $u_{n}$ of polynomials $f_{n}(z)=z^{p^{n}}-\left(x_{n}+t^{p^{n}}\right), n \geq 1$, and $u_{n}^{\prime}$ of polynomials $g_{n}(z)=f_{n}(z)-p t$, $n \geq 1$, respectively. Finally, let $K$ and $K^{\prime}$ be the completions of $K_{1}^{\prime}$ and $K_{1}^{\prime \prime}$ respectively. Since $K$ and $K^{\prime}$ have the same residue fields there is an analytically isomorphic $K \rightarrow K^{\prime}$ which leaves residues fixed [9, Corollary 1, p. 431]. Let $K_{0}^{\prime}$ be the image of $K_{0}$.

A.1 Proposition. $K_{0}$ is algebraically closed in $K$ and in $K^{\prime}$.

Proof. By Lemmas 3.3, 3.4 and Corollary 3.5 it will suffice to show that for any $n, \bar{b}_{n}=\bar{x}_{n}+\bar{t}^{p^{n}}$ does not satisfy the condition

$$
\bar{b}_{n}^{p^{n}}=\left(\bar{a} \bar{c}_{n}\right)^{p^{n}}
$$

with $\bar{a} \in k_{0}, \bar{c}_{n} \in k_{0}\left(\bar{t}, \bar{u}_{1}, \cdots, \bar{u}_{n-1}\right)$ and $0 \leq m<n$. For if $\bar{a}$ and $\bar{c}_{m}$ exist fulfilling A.2 then $\bar{c}_{n}^{p^{n}}$ is a rational function over $k^{*}$ in $\tau^{p^{n}}$, the $\bar{x}_{i}^{p^{n}}$ and $\bar{u}_{1}^{p^{n}}, \cdots, \bar{u}_{n+1}^{p^{n}}$ whereas $\bar{a}$ is a rational expression in the $\bar{x}_{i}$ over $k^{*}$. Since $\bar{u}_{i}^{p^{n}}=\bar{x}_{i}^{p^{n-i}}+\left(\bar{t}^{p^{i}}\right)^{p^{n-i}}, \bar{c}_{n}^{p^{n}}$ is a rational expression in $\bar{t}^{p^{n}}$ and the $\bar{x}_{i}$. Since $\bar{b}^{p^{n}}=\bar{x}_{n}^{p^{n}}+\bar{t}^{p^{m+n}}=\bar{a}^{p^{n}} \bar{c}_{n}^{p^{n}}$ we may assume $\bar{a}^{p^{n}}$ is a polynomial in the $\bar{x}_{i}$ over $k^{*}$ and $\bar{c}_{n}^{p^{n}}$ is a polynomial $\bar{t}^{p^{n}}$ and the $\bar{x}_{i}$. Comparing coefficients of $\bar{t}^{p^{m+n}}$ we conclude that $\bar{a}^{p^{n}} \in k^{*}$. We note next that $\bar{x}_{n}$ appears in $\bar{c}_{n}$ only in powers of $p^{n}$. Thus the equality A.2 cannot be obtained.

A.3 Proposition. $K_{0}$ is the field of constants of an integral derivation.

Pruof. Define $d \in \operatorname{Der}\left(K / K_{0}\right)$ by the condition $d(t)=1$. Then

$$
d\left(u_{n}\right)=-f_{n}^{d}(u) / f_{n}^{\prime}(u)=p^{n} t^{p^{n}-1} / p^{n} u^{p^{n}-1} \text {. }
$$

Since each integer in $K_{1}^{\prime}$ is a polynomial in the $u_{i}$ with coefficients which are integers in $K_{0}$ it follows that $\left.d\right|_{K_{1}}$ is integral. Hence $d$ is integral. The field of constants of $\left.d\right|_{K_{1}} ^{\prime}$ is $K_{0}$ since $K_{1}=K_{0}(t)$ and $d(t)=1$. Thus, since $K_{0}$ is algebraically closed in $K, K_{0}$ is the field of constants of $d$ by Lemma 2.0 .

A.4 Proposition. There does not exist an integral derivation on $K$ with field of constants $K_{0}^{\prime}$.

Proof. It will suffice to prove there is no integral derivation $d^{\prime}$ on $K^{\prime}$ with field of constants $K_{0}$ in view of the analytic isomorphism used to define $K_{0}^{\prime}$. Suppose there is such a derivation $d^{\prime}$. Then $d^{\prime}(t) \neq 0$ and $d^{\prime}\left(x_{n}\right)=0$ for all $n>0$. Let $V\left(d^{\prime}(t)\right)=m$. Then 
$V\left(d^{\prime}\left(u_{m+2}\right)\right)=V\left(g_{m+2}^{d^{\prime}}\left(u_{m+2}\right) / g_{m+2}^{\prime}\left(u_{m+2}\right)\right)=V\left(p^{m+2} d^{\prime}(t)+p d^{\prime}(t)\right)-V\left(p^{m+2}\right)=-1$ contradicting the assumption that $d^{\prime}$ is integral.

We observe finally that since $\bar{u}_{n}^{p^{n}}=\bar{x}_{n}+\bar{t}^{p^{n}}, \bar{u}_{n}-\bar{t}$ is a $p^{n}$ th root of $\bar{x}_{n}$ and hence $k_{0}$ is not algebraically closed in $k_{\text {. }}$

Example 2.

Summary. Fields $k_{0} \subset k$ of characteristic $p$ are constructed such that if $K_{0} \subset K$ are $p$-adic fields with these as residue fields there is no nonzero analytic derivation on $K$ with field of constants containing $K_{0} \cdot k_{0}$ is algebraically closed in $k$ and contains the maximal perfect subfield of $k$. This is an adaptation of an example due to Mac Lane [10, pp. 36-37].

Construction. Let $k^{*}$ be a perfect field of characteristic $p \neq 0$. Let $\bar{X}=$ $\left\{\bar{x}_{1}, \bar{x}_{2}, \ldots\right\}$ be a denumerable set and $\bar{t}$ be such that $\bar{X} \cup\{\bar{t}\}$ is algebraically independent over $k^{*}$. Let $\bar{U}=\left\{u_{i}\right\}_{i \geq 1}$ where $\bar{u}$ is a root of $\bar{f}_{1}(z)=z^{p}$ $\left(\bar{x}_{1}+\bar{t}\right)$ and for $n>1, u_{n}$ is a root of $\bar{f}_{n}(z)=z^{p}-\left(\bar{x}_{n}+\bar{u}_{n-1}\right)$. Mac Lane has shown that $k_{0}=k^{*}(\bar{X})$ is algebraically closed in $k=k_{0}(\bar{t}, \bar{U})$, that $\bar{X}$ is a $p$ basis for $k$, and that $k^{*}$ is the maximal perfect subfield of $k$.

A.5 Proposition. Let $K_{0} \subset K$ be $p$-adic fields with residue fields $\ldots, \subset k$ respectively. There is no analytic derivation on $K$ with $K_{0}$ in its field of constants.

Proof. If $d \in \operatorname{Der}(K)$ has $K_{0}$ in its field of constants then multiplying by a suitable power of $p$ we can assume that $d$ induces a nontrivial derivation on $k$ which is constant on $k_{0}$. However, since $k_{0}$ contains a $p$-basis for $k_{\text {, }}$ $\operatorname{Der}\left(k / k_{0}\right)=\{0\}$. Hence $\operatorname{Der}\left(K / K_{0}\right)=\{0\}$.

Example 3.

Summary. We construct $p$-adic fields $K_{0} \subset K$ with residue fields $k_{0} \subset k$ such that every $\delta$ in $\operatorname{Der}\left(k / k_{0}\right)$ is induced by some $d$ in $\operatorname{Der}\left(K / K_{0}\right)$ yet $k$ is not separable over $k_{0} \cdot k_{0}$ contains $k^{*}$ the maximal perfect subfield of $k_{\text {. }}$

Construction. Let $k^{*}$ be a perfect field of characteristic $p \neq 0$. Let $\bar{X}=$ $\left\{\bar{x}_{0}, \bar{x}_{1}, \bar{x}_{2}, \ldots\right\}$ and $\bar{Y}=\left\{\bar{y}_{1}, \bar{y}_{2}, \ldots\right\}$ be denumerable sets and let $\bar{t}$ be such that $\bar{X} \cup \bar{Y} \cup\{\bar{t}\}$ is algebraically independent over $k^{*}$. Let $\bar{u}_{0}$ be a root of $\bar{f}_{0}(z)=z^{p}-\left(\bar{x}_{0}+\bar{t}^{p}\right)$ and, for $n>0$, let $\bar{u}_{n}$ be a root of $\bar{f}_{n}(z)=z^{p}-\left(\bar{x}_{n}+\right.$ $\left.\bar{u}_{n-1}\right)$. Also, $\bar{v}_{1}$ is a root of $\bar{g}_{1}(z)=z^{p}-\left(\bar{y}_{1}+\bar{t}\right)$ and for $n>1, \bar{v}_{n}$ is a root of $\bar{g}_{n}(z)=z^{p}-\left(\bar{y}_{n}+v_{n-1}\right)$. Define $k_{0}$ to be $k^{*}(\bar{X}, \bar{Y})$ and $k=k_{0}(\bar{t}, \bar{U}, \bar{V})$ where $\bar{U}=\left\{u_{i} \mid i=0,1, \cdots\right\}$ and $\bar{V}=\left\{v_{i} \mid i=1,2, \cdots\right\}$.

A.6 Contention. $k=k_{0}\left(k^{p}\right)$ and bence $\operatorname{Der}\left(k / k_{0}\right)=\{0\}$.

Proof. The following equalities establish that $\{\bar{t}\} \cup \bar{U} \cup \bar{V} \subset k_{0}\left(k^{p}\right): \bar{t}=$ $\bar{v}_{1}^{p}-\bar{y}_{1}, \bar{u}_{n}=\bar{u}_{n+1}^{p}-\bar{x}_{n}$ for $n \geq 0$, and $\bar{v}_{n}=\bar{v}_{n+1}^{p}-\bar{y}_{n}$ for $n \geq 1$. 
We show next that $k^{*}$ is in fact the maximal perfect subfield as follows.

A.7 Contention. For each $n \geq 0$ the set $W_{n}=\{\bar{t}\} \cup\left\{\bar{x}_{m} \mid m \geq n\right\} \cup\left\{\bar{y}_{m} \mid m\right.$ $\geq n\} \cup\left\{\bar{u}_{m} \mid m<n\right\} \cup\left\{\bar{v}_{m} \mid m<n\right\}$ is algebraically independent over $k^{*}$.

Proof. $\bar{W}_{0}=\bar{X} \cup \bar{Y} \cup\{\bar{t}\}$ which is algebraically independent by construction. Assume, $\bar{W}_{n}$ algebraically independent over $k^{*}$ and consider any nontrivial algebraic relation in $\bar{W}_{n+1}$. Taking the pth power followed by the substitutions $\bar{u}_{n}^{p}=\bar{x}_{n}+\bar{u}_{n-1}, \bar{v}_{n}^{p}=\bar{y}_{n}+\bar{v}_{n-1}$ yields a nontrivial algebraic relation in $\bar{W}_{n^{\prime}}$ a contradiction.

By Contention A.7 we see that $\bar{U} \cup \bar{V} \cup\{\bar{t}\}$ is algebraically independent over $k^{*}$. Since $\bar{x}_{0}=\bar{u}_{0}^{p}-\bar{t}^{p} ; \bar{x}_{n}=\bar{u}_{n}^{p}-\bar{u}_{n-1}$, for $n \geq 1$ it follows that $k=$ $k^{*}(\bar{U}, \bar{V}, \bar{t})$. It follows that $k^{*}$ is the maximal perfect subfield of $k$.

Note finally that by definition of $\bar{u}_{0}$ we have $\bar{x}_{0}=\left(\bar{u}_{0}-\bar{t}\right)^{p}$. Thus $\bar{x}_{0}$ is $p$-independent in $k_{0}$ but is not $p$-independent in $k$. It follows that $k$ is not separable over $k_{0}$.

\section{BIBLIOGRAPHY}

1. R. Baer, Algebraische Theorie der differentierbaren Funktionenkörper, S.-B. Heidelberger Akad. Wiss. Abh. 8 (1927), 15 - 32.

2. N. Heerema, Exponential automorphisms of complete local rings, Math. Z, 122 (1971), 299-306.

3. - Derivations and embeddings of a field in its power series ring, Proc. Amer. Math. Soc. 11 (1960), 188 - 194. MR 23 \# A893.

4. —_, Derivations on p-adic fields, Trans. Amer. Math. Soc. 102 (1962), 346 351. MR $26 \# 1311$.

5. - Inertial automorphisms of a class of wildly ramified v-rings, Trans. Amer. Math. Soc. 132 (1968), 45-54. MR 36 \#6407.

6. - Convergent higher derivations on local rings, Trans. Amer. Math. Soc. 132 (1968), 31 -44. MR 36 \#6406.

7. N. Jacobson, Lectures in abstract algebra. Vol. III: Theory of fields and Galois theory, Van Nostrand, Princeton, N. J., 1964. MR 30 \#3087.

8. S. Mac Lane, The uniqueness of the power series representation of certain field with valuations, Ann. of Math. (2) 39 (1938), 370 - 382.

9. - Subfields and automorphisms groups of p-adic fields, Ann. of Math. (2) 40 (1939), $423-442$.

10. - Steinitz field towers for modular fields, Trans. Amer. Math. Soc. 46 (1939), 23-45. MR 1, 3.

11. J. Neggers, Derivations on $\bar{p}$-adic fields, Trans. Amer. Math. Soc. 115 (1965), 496-504. MR $33 \# 5610$.

12. T. Peter, An intrinsic characterization of those derivations on the residue field of a ramified v-ring which lift, Dissertation, Florida State University, Tallahassee, Fla., 1973.

DEPARTMENT OF MATHEMATICS, STETSON UNIVERSITY, DELAND, FLORIDA 32720 FLORIDA 32306

DEPARTMENT OF MATHEMATICS, FLORIDA ST ATE UNIVERSITY, TALLAHASSEE, 